\title{
Revisiones
}

\section{Acreditación de Comités de Ética en Investigación, como parte de un proceso}

\author{
Duilio Fuentes ${ }^{1,2}$, Diana Revilla ${ }^{3}$
}

Resumen

Palabras clave

\begin{abstract}
Los comités de ética en investigación tienen un rol importante en la protección del bienestar y seguridad de los participantes; y, en países donde la población es potencialmente vulnerable a la explotación, su presencia es de gran importancia. Ante este hecho, y tomando en cuenta la existencia de graves problemas y la necesidad de reformar el sistema de protección de los sujetos de investigación, se plantea la necesidad de compartir estándares de calidad y criterios de evaluación adecuados y homogéneos, creándose así el término ‘acreditación'. Por lo tanto, debemos situarnos en la realidad local, y a raíz de ello poder entender el significado de la acreditación de los comités de ética en investigación como un proceso, justificando su ejecución y analizando las fortalezas y debilidades de que la realice una entidad gubernamental.

Investigación; comités de ética; ética; acreditación.
\end{abstract}

\begin{abstract}
Acreditation of Research Ethics Committees, as part of a process

Abstract

The research ethics committee plays an important role in the protection, wellbeing and security of research participants. This role is more important in countries where the population has the potential of being vulnerable to exploitation. In presence of this fact and considering the existence of serious problems with the necessity of subjects protection system reform, the need of shared quality standards and appropriate criteria is posed, creating the term 'accreditation'. Knowing local reality and trying to understand the meaning of accreditation of research ethics committee as a process, its execution is justified and a strength/ weakness analyses is done in the event it is performed by a governmententity.
\end{abstract}

Key words: Research; ethics committees; ethics; accreditation.

Los Comités de Bioética pueden ser definidos como grupos de consenso o estructuras de diálogo y decisión bioética que asumen la responsabilidad de intentar clarifi-

1 Médico de Medicina Interna. Universidad Nacional Mayor de San Marcos. Lima, Perú.

Instituto Nacional de Salud. Lima, Perú.

3 Médico Cirujano. Universidad Peruana Cayetano Heredia. Lima, Perú. car y resolver conflictos de valores que se presentan en la investigación o en la práctica clínica.

Los Comités de Ética en investigación existen para proteger el bienestar y seguridad de los participantes en la investigación y evaluar el balance riesgo/beneficio de un estudio, dentro del contexto de un protocolo de investigación. Asimismo, realizan una evaluación del rigor científico y de factibilidad del estudio.

En países de América Latina, donde un segmento considerable de la población se presenta como potencialmente vulnerable a la explotación, debido a factores como la pobreza, el escaso acceso a servicios de salud y el bajo nivel de instrucción, la presencia de los Comités de Ética en Investigación es de gran importancia.

Observando este panorama, el objetivo de este trabajo es tratar de responder a estos cuestionamientos: ¿Cuál es la realidad de los Comités de Ética en investigación 
(CEI) en América Latina, y particularmente en el Perú, a diferencia de los países desarrollados? Escuchamos en varios cursos, conferencias, foros latinoamericanos, artículos y regulaciones sobre ética en investigación y, en particular al tratar la problemática de los CEI, sobre acreditación de los mismos. ¿Qué significa este término? ¿Por qué deberían ser realizados? Y si es que se realizara, ¿quién debería hacerlo?

\section{Desarrollo y realidades diferentes: Estados Unidos, Europa y América Latina}

Los Comités de ética en investigación, conocidos en los EE UU bajo la denominación genérica de Institutional Review Board $\left({ }^{1}\right)$ (IRB, textualmente, comités institucionales de revisión) entraron en vigor en el año 1974, cuando el Congreso Norteamericano estableció que estos comités debían evaluar la investigación sobre sujetos humanos financiada con fondos federales. Esta regulación fue consecuencia de la difusión en la prensa de que se habían realizado investigaciones en fetos humanos con el respaldo financiero del National Institute of Health. El Congreso Norteamericano nombró por Ley 93-348, una comisión para la protección de los seres humanos (Commission for the Protection of Human Subjects of Biomedical and Behavioral Research), para establecer los principios éticos básicos sobre los que debía conducirse la investigación biomédica.

Los Comités de Ética en EE UU se encuentran bajo la regulación de la Oficina Federal para la Protección de la Investigación con Sujetos Humanos (Office for human Research Protections-OHRP), para la realización de todos los proyectos de investigación. El Departamento de Salud y Servicios Humanos (DHHS) de los EE UU destacó en un informe la existencia de graves problemas y la necesidad de reformar el sistema de protección de los sujetos de investigación $\left(^{2}\right)$. En EE UU y Canadá, el siste- ma de acreditación de comités de ética en investigación es voluntario y dirigido por una instancia no gubernamental.

La legislación sobre Ensayos Clínicos en la Unión Europea aparece en 1991, con una directriz: "Normas de Buena Práctica Clínica para ensayos clínicos en la Comunidad Europea" (documento III/3976/88), en donde puntualiza la protección de los sujetos en investigación y el rol de los Comités de ética. La Agencia Europea del Medicamento (EMEA) funciona desde 1995. En el 2001, el Parlamento Europeo promulga la Directiva 2001/20/CE, cuyo objetivo es armonizar las normas científicas y racionalizar los procedimientos documentales y administrativos utilizados en la realización de los ensayos clínicos con medicamentos de uso humano. La norma establece los principios que deben ser aplicados para la protección de los sujetos del ensayo, los requisitos adicionales para los ensayos clínicos con menores de edad y adultos incapacitados; determina que en todos los ensayos clínicos deben cumplirse las normas de buenas prácticas clínicas (BPC); condiciona la realización del ensayo al dictamen favorable previo de un comité de ética en investigación clínica (CEIC), así como a la autorización administrativa previa de la autoridad sanitaria competente.

En España, la RD 223/2004 creó el dictamen único, a cargo del Centro Coordinador de Comités Éticos de Investigación Clínica. Dicha organización se constituye como la unidad técnica operativa que tiene como objetivo facilitar que los CEIC acreditados por las comunidades autónomas puedan compartir estándares de calidad y criterios de evaluación adecuados y homogéneos y favorecer la agilidad en el proceso de obtención del dictamen único.

Los Comités Éticos de Investigación Clínica serán acreditados por la autoridad sanitaria competente en cada comunidad autónoma, quien determinará el ámbito geográ- 
fico e institucional de cada comité. Dicha acreditación deberá ser renovada periódicamente por dicha autoridad sanitaria según los procedimientos y plazos que ésta determine. Tanto la acreditación inicial como sus renovaciones deberán ser notificadas a la Agencia Española de Medicamentos y Productos Sanitarios y al Centro Coordinador de Comités Éticos de Investigación Clínica.

En América Latina, durante los últimos 10 años se ha incrementado el número de pacientes latinoamericanos que participan en estudios clínicos. Argentina, Brasil y México se encuentran entre los países con más investigaciones, seguido en un segundo plano por Chile, Perú y Colombia. Como resultado, aumentó el número de centros de investigación, de Organizaciones de Investigación por Contrato (CRO) y de CEIC.

En Brasil $\left({ }^{3}\right)$, en 1988, el Consejo Nacional de Salud (CNS), del Ministerio de Salud, determinó que toda institución de salud que realiza investigaciones en seres humanos tuviese un Comité de Ética. El CNS determinaba: «la investigación solamente podrá ser iniciada después de la opinión favorable, por escrito, del Comité de Ética y del Comité de Seguridad Biológica, conforme el caso». En 1996, después de un exhaustivo trabajo de revisión de la Resolución de 1988, el CNS publicó la Resolución 196/ 96, determinando que cualquier investigación con seres humanos, no solo en las áreas biomédicas, deben ser aprobadas por un Comité de Ética en investigación. Esta Resolución establece las normas que serán seguidas por los Comités en su organización, atribuciones y procedimientos.

En Argentina*, el Ministerio de Salud, en 1985, encargó a una Comisión de reconocidos profesionales la redacción de las normas que iban a regir las investigaciones clínicas en la Argentina. Esta Comisión dio

* Información de Martín Seoane y Ricardo Bolaños, profesionales que se desempeñan en la ANMAT. forma a lo que se publica como: "Normas para solicitar autorizaciones para realizar estudios e investigaciones de Farmacología Clínica". Se hace mención también a las disposiciones de la OMS en los aspectos científicos, éticos y jurídicos de los ensayos en seres humanos. Esta disposición se convirtió en un elemento simbólico. Luego de la creación de la Administración Nacional de Medicamentos, Alimentos y Tecnología Médica (ANMAT), se puso en vigencia la Disposición 4854, en 1996, que reafirmaba los aspectos éticos, incluidos en la declaración de Helsinki y afianzaba el concepto de autorizaciones expresas de los Comités de Docencia e Investigación y de un Comité de Ética independiente. Luego, la ANMAT sancionó la Disposición 5330, en 1997, que es la actual normativa vigente en Argentina. Actualmente, se ha elaborado un proyecto de ley, que se encuentra en el Congreso de la República Argentina, con el objeto de normar el funcionamiento de los Comités de Ética en investigación en Salud en todo el territorio nacional, creando para ello la Dirección Nacional de Registro, Acreditación y Supervisión de los Comités de Ética de la Investigación en Salud.

En Colombia $\left({ }^{4}\right)$, se creó la Comisión Intersectorial de Bioética en el año 2000, órgano consultivo del gobierno para estudio y definición de políticas en temas relacionados con la investigación y la respectiva protección del ser humano. En muchas instituciones se han organizado comités de bioética y existe una regulación que obliga a establecer comités éticos para la experimentación clínica (Resolución 8430, de 1993).

En Chile ( ${ }^{5}$ ), el año 1997, el Ministerio de Salud convocó a un grupo de expertos al estudio de los diferentes códigos existentes en el mundo, con el fin de regularizar la ejecución de ensayos clínicos que utilizan medicamentos en seres humanos. $\mathrm{Si}$ bien es cierto, en alguna medida existían normativas, como la reglamentación de los Servicios de Salud respecto a la autoriza- 
ción que los directores de hospitales deben dar a los proyectos de investigación y a la creación de Comités de Ética, para la supervisión de éstos. El año 2001, el Ministerio de Salud capacitó y acreditó en Chile los primeros Comités de Ética Científicos, cuyo propósito es contribuir a salvaguardar la dignidad, los derechos, la seguridad y el bienestar de todos los participantes actuales y potenciales de la investigación.

En el Perú $\left({ }^{6}\right)$, se estableció en 1982, según Decreto Supremo No 0212-82-SA, las "Normas para el uso de drogas en los ensayos clínicos", donde se establecía que el desarrollo de las investigaciones se realice en el marco de las normas internacionales de ética en investigación (Declaración de Helsinki). Las estadísticas nacionales, publicadas por el Instituto Nacional de Salud, desde el año 1995 hasta la fecha, muestran un incremento anual sostenido del número de estudios clínicos aprobados. Este crecimiento se correlaciona con el aumento progresivo de centros de investigación, investigadores y comités de ética en investigación en Lima y otros departamentos. El Ministerio de Salud, por intermedio del Instituto Nacional de Salud (entidad reguladora desde el año 2003), asumió su rol regulador y elaboró una Norma nacional recientemente publicada (Decreto Supremo No 017-06-SA). En esta norma se establece, además de todos los aspectos regulatorios para la presentación de ensayos clínicos, el rol de los Comités de Ética en investigación, considerando pautas para su composición, funcionamiento y registro a la autoridad reguladora.

\section{Problemática de los Comités de Ética en Investigación en América Latina}

Vimos en el parágrafo anterior que el desarrollo de los CEI en los países de América Latina no ha sido parejo, sino que fue adaptándose de una forma variable en los distintos países. Según un estudio de la Unidad de Bioética de la OPS/OMS, entre los años 1999 y $2002\left({ }^{7}\right)$, y los identificados en nuestra experiencia, podríamos señalar los siguientes problemas:

1) El control ético de los estudios no es entendido o valorado por la mayoría de los investigadores. Tiende a ser considerado como una carga burocrática o como una instancia de poder.

2) En América Latina, la mayor parte de la investigación es multicéntrica y llevada a cabo por instituciones del extranjero. Existe la convicción en algunos actores que conducen la investigación de que los protocolos de investigación externa no necesitan análisis local.

3) Falta de diferenciación entre los comités de evaluación ética de la investigación y los comités de ética hospitalaria. En muchas ocasiones, los comités de ética hospitalaria -que se han ido constituyendo en establecimientos de atención de salud- han asumido sin mucha preparación ni experiencia la evaluación de protocolos de investigación clínica.

4) Falta de financiación y capacidad administrativa para llevar a cabo las actividades que deben realizar los comités de evaluación ética. Las instituciones donde se forman dichos comités no ofrecen el apoyo logístico adecuado (secretariado, archivos que mantengan la confidencialidad, base de datos, etc).

5) La multiplicidad de funciones de los miembros del CEI -asistencial, administrativos, gerencial y docencia- y esto sumado a la falta de apoyo institucional, conlleva a que las funciones y responsabilidades que debe cumplir el CEI no sean satisfechas.

6) Específicamente en el Perú, existe una centralización de los CEI, principalmente en la capital del país, pese a que cada vez es más frecuente la ejecución de estudios en diferentes regiones. Es importante considerar este tema, ya que existen universidades en estas regiones que forman profesionales y que muy bien 
podrían asumir esta labor, tomando en cuenta las particularidades culturales, costumbres y condiciones locales.

7) Muchos CEI no cuentan con Manual de Procedimientos Operativos (POEs) que especifiquen su conformación, funciones de los miembros, procedimientos de desempeño, relaciones y manejo de conflicto de intereses.

8) Falta un proceso formal de calendarización de reuniones. Algunas se reúnen esporádicamente o cuando son específicamente requeridos.

9) Se ha evidenciado la dificultad que existe en cuanto al perfil que debe tener la persona elegida como miembro de la comunidad de los CEI.

10) No todos los miembros de los comités de evaluación ética conocen completamente su función. Muchos lo asumen, principalmente en Comités de Ética Institucional, como defensores incondicionales de los intereses de las instituciones que representan, olvidándose de los intereses de los sujetos en investigación, no manejando la presión de ciertos conflictos de interés secundarios, como económicos (para la institución o servicio donde se desempeñan), sociales (no romper la amistad del investigador principal o sus colegas) o profesionales (futuras convocatorias a estudios similares).

11) Los CEI externos o no institucionales y los comités que pertenecen a una institución privada o no gubernamental que se dedica a la investigación, son financieramente dependientes de actores comerciales que debieran controlar. Es razonable la presunción que los intereses financieros pueden tener un impacto consciente o inconscientemente a favor de los intereses de estas instituciones, pudiéndose observar, por ejemplo, en lo expeditivo de sus aprobaciones.

12) Existe falta de capacitación en ética en investigación, por lo que se crea la necesidad de capacitar a profesionales que conformen Comités de Bioética de la Investigación.

13) Falta de supervisión y mecanismos de control para informar sobre efectos adversos en muchas de las investigaciones que se realiza en América Latina.

14) Falta de seguimiento y 'monitoreo ético' de las investigaciones aprobadas por los CEI.

15) En los estudios multicéntricos, existe una variedad, que se hace evidente por extremos muy marcados, en cuanto al tiempo de revisión y de expedición de opinión. Se evidencia, asimismo, la falta de acuerdo de algunos CEI en cuanto a sus correcciones, las cuales se realizan en 2 o más cartas, haciendo la comunicación con el investigador principal interminable y engorrosa.

16) Falta de comunicación entre los CEI y la autoridad reguladora. Esto trae como consecuencia que sea incierto el destino que siguen las investigaciones desaprobadas por los CEI y propicie el IRB shopping.

17) Las normativas nacionales que regulan la investigación en seres humanos en los países de América Latina son escasas, en pocos casos inexistentes y en otros recién se están desarrollando; principalmente, a la regulación ética de las investigaciones.

18) Existen pocos Comités Nacionales que supervisen el trabajo de los comités locales y la investigación en general.

\section{¿Por qué debería realizarse la acreditación de CEI?}

Para quienes desarrollan programas de Sistemas de Calidad, como ISO (International Organization for Standaridization) y BARQA (British Association for Research Quality Assurance), "acreditación es un procedimiento mediante el cual un organismo autorizado da reconocimiento formal de que 
una organización o individuo es competente para llevar a cabo tareas específicas".

Observando la problemática de los CEI en nuestra región, se crea la necesidad de estandarizar y establecer un mecanismo de acreditación que sea transparente y propio de cada realidad, ya que, como vimos antes, el contexto de creación y desarrollo de los CEI es distinto en cada país.

Bajo esta premisa, la acreditación es importante porque:

1. Estandariza los procesos de revisión, generando eficiencia en el desempeño de los CEI.

2. Certificaría la idoneidad, conocimiento y seriedad de los integrantes de los CEI, lo cual potenciaría el resguardo de los derechos e intereses de los sujetos en investigación.

Asimismo, sería un instrumento para asumir responsabilidades y funciones, debido a que:

1. Las autoridades de las instituciones donde se realiza la investigación, que en su mayor parte son establecimientos públicos, percibirían la importancia y necesidad de constituir CEI idóneos, con el adecuado apoyo administrativo y logístico. Asimismo, asumirían la trascendencia de lo que significa la investigación en salud en las instituciones que dirigen; introduciendo políticas institucionales que direccionen la apropiada independencia de los CEI y creando una cultura de ética en investigación en los investigadores y profesionales de salud en general.

2 . Establece procedimientos en la composición y miembros del CEI en cuanto a requerimientos establecidos en el manejo y transparencia de conflicto de intereses.

Por lo tanto, podríamos citar algunas razones para su implementación:

1. Los mecanismos idóneos y transparentes de los CEI pueden servir como instru- mento para crear confianza pública en la investigación, desechando paulatinamente la idea de que los sujetos en investigación son 'conejillos de indias' y creando mayor conciencia ciudadana sobre la relación entre la ciencia y la sociedad.

2. En el contexto de la globalización, aumentaría la confianza en los patrocinadores y facilitaría la oportunidad de investigaciones colaborativas.

3. Podría propiciar la formación de organismos o instituciones que funcionen como Red de CEI nacionales, que se conviertan en foros de discusión de situaciones éticas controversiales en el campo de la investigación. Como parte de un proceso, estos organismos establecerían estándares comunes de actuación, previa discusión y consenso de sus miembros, creando políticas de manejo en la solución de aquellas controversias y liderando la formación bioética en toda la comunidad científica.

\section{¿Quién debe estar a cargo de la conducción de la acreditación?}

Viendo nuevamente el desarrollo de los CEI en diferentes regiones, se observa que existen 2 situaciones en países desarrollados: En Estados Unidos y Canadá, la acreditación es voluntaria y la realiza un organismo no gubernamental, situación diferente a la de Europa, específicamente España, donde la acreditación es requisito fundamental para la evaluación de ensayos clínicos y es dirigida por un organismo gubernamental.

\section{¿Qué podemos decir del sistema no gubernamental?}

\section{Fortalezas}

1) Las organizaciones no gubernamentales pueden ser consistentes y estables en el tiempo, no afectadas por cambios o políticas gubernamentales. 
2) Pueden generar un presupuesto propio y libre de dependencias gubernamentales, que pueden variar según cambios políticos.

3 ) Establecer procesos más expeditivos libre del sistema burocrático de los Estados.

\section{Debilidades y amenazas}

Este sistema puede conllevar a conflicto de intereses financieros y de poder, que pueden amenazar la transparencia en el proceso de acreditación, más aún si no existe un sistema que asegure la calidad del proceso.

El sistema europeo tiene la ventaja que, desde el establecimiento de la Comunidad Europea, trajo muchos beneficios a los países miembros, propiciando su desarrollo como macrorregión en el aspecto económico, político y social. Este sistema, más aún en España, fortaleció el Estado e incentivó la descentralización y autonomía de diferentes regiones, resultando en una gran fortaleza para el sistema de acreditación gubernamental.

En América Latina, la situación tiende a que la acreditación la realice un estamento gubernamental. En Chile, el Ministerio de Salud acredita los CEI y la revisión es centralizada, mientras que en Argentina existe un proyecto de registro, acreditación y seguimiento de CEI en el ámbito nacional, dirigido por una institución gubernamental.

En el Perú, con la aprobación de la nueva reglamentación, se establece el registro de los CEI bajo una instancia gubernamental (el Instituto Nacional de Salud).

\section{Bajo estas premisas, ¿Qué podemos decir de este sistema?}

\section{Fortalezas}

1) Dentro de la misión de los estados y respectivos ministerios, está la de protec- ción de los derechos de las personas y la promoción de su bienestar; por lo que esta misión es inherente y concordante con el objetivo fundamental de la acreditación de los CEI.

2 ) Los Estados latinoamericanos, y en particular el Perú, promueven, mediante la creación de instituciones gubernamentales, la investigación en salud. Estas dependencias del Estado manejan un presupuesto que bien podría invertirse en el desarrollo de este proceso de registro y acreditación de CEI.

\section{Oportunidad}

En el Perú, están en pleno desarrollo tanto la bioética como disciplina como la conformación de los CEI, en instituciones públicas y privadas. Asimismo, el Estado (mediante el INS), como parte de este proceso, ha comenzado a ejercer su rol regulador en la investigación clínica en el ámbito nacional, elaborando una norma que establece las pautas a seguir por todos los actores que intervienen en la investigación, y dentro de este proceso es que ha establecido el registro de los CEI en el ámbito nacional. Iniciado el camino, es una oportunidad para poder continuar el proceso.

\section{Debilidades y amenazas}

Los gobiernos y políticas de los Estados latinoamericanos no son consistentes y estables en el tiempo, lo que resulta en una debilidad para desarrollar procesos adecuados, transparentes y continuos, principalmente en este tema de la acreditación. Una constante en nuestros gobiernos son los sistemas tan burocráticos, así como la dirección en la conducción de las dependencias gubernamentales, que está a cargo de funcionarios públicos elegidos en forma arbitraria y que no guardan el perfil apropiado, por lo que resultaría una amenaza para que no puedan cumplirse estos procesos. 


\section{Conclusiones}

Concluiremos estableciendo la necesidad de implementar la acreditación de los CEI, particularmente en el Perú, como parte de un proceso que sea factible y adecuado según nuestra propia realidad.

Por lo tanto, este proceso debe cumplir los siguientes pasos:

1) Identificar y establecer estándares regulatorios propios.

2) Establecer un proceso continuo y dinámico:

a) Registro.

b) Inspecciones.

c) Acreditación.

d) Programa de capacitación continua para los miembros del CEI.

e) Evaluación periódica.

f) Cambio de exigencias en el tiempo.

g) Evaluación y seguimiento continuo.

Los encargados de la conducción de este proceso deben cumplir con:

1) Educación continua.

2) Financiamiento y administración transparentes.

\section{Agradecimiento}

A la Dra. Florencia Luna, directora del programa de Bioética de la Facultad Latinoamericana de Ciencias So- ciales (FLACSO) - Argentina, por sus sugerencias y revisión del artículo.

\section{REFERENCIAS BIBLIOGRÁFICAS}

1. López MM, Batista-Miranda JE. Comités éticos de investigación: una compilación necesaria. Med Clin (Barc). 1995;105(8):298-301.

2. Luna F. Comités de ética de investigación: Y por casa... ¿cómo andamos?. México: FLACEIS; 2002.

3. Hardy E, Ferreira S, Duarte MJ, Hebling EM. Comitês de Ética em pesquisa: adecuaçäo à resoluçäo 196/96. Rev Assoc Med Bras. 2004;50(4):457-62.

4. Manrique JI. Generalidades de los Comités de Bioética y su utilidad como medio probatorio en los procesos judiciales y éticos. Médico Legal. 2003 enero-marzo:64-71.

5. Acevedo I. Aspectos éticos en la investigación científica. Ciencia y Enfermería. 2002;8(1):15-8.

6. Lecca L, Llanos-Zavala F, Ignacio E. Características de los Comités de Ética en Investigación del Perú autorizados para aprobar ensayos clínicos. Rev Med Hered. 2005;16(1):310.

7. World Health Organization. DGO/ETH and Regional Focal Points [videoconference]. 2003 May 6th. Geneva: WHO; 2003.

Manuscrito recibido el 07 de febrero de 2007 y aceptado para publicación el 19 de marzo de 2007.

Correspondencia:

Duilio Jesús Fuentes Delgado

Av. Del Parque Norte 332 tercer piso, Córpac

Lima 27, Perú

Correo-e:jediaqp@yahoo.com 